Proyecciones Journal of Mathematics

Vol. 31, No 4, pp. 363-372, December 2012.

Universidad Católica del Norte

Antofagasta - Chile

\title{
Equitable Graph of a Graph
}

\author{
Kuppusamy Markandan Dharmalingam
}

The Madura College, India

Received : April 2012. Accepted : September 2012

\begin{abstract}
$A$ subset $D$ of $V$ is called an equitable dominating set if for every $v \in V-D$ there exists a vertex $u \in D$ such that $u v \in E(G)$ and $|d(u)-d(v)| \leq 1$, where $d(u)$ denotes the degree of vertex $u$ and $d(v)$ denotes the degree of vertex $v$. The minimum cardinality of such a dominating set is denoted by $\gamma^{e}$ and is called the equitable domination number of G.Degree Equitable domination on Graphs were introduced in [10].This Paper aims at the study of a new concept called equitable graph of a graph introduced by Prof. E. Sampathkumar. In this paper we show that there is relation between $\gamma\left(G^{e}\right)$ and $\gamma^{e}(G)$. Further results on the new parameter $\gamma\left(G^{e}\right)$ are discussed.
\end{abstract}

Keywords : Degree Equitable Number and Equitable Graph of a Graph.

2011 Mathematics Subject Classification : Primary. 05C. 


\section{Introduction and Definitions}

New concepts of domination arise from practical considerations. In a network, nodes with nearly equal capacity may interact with each other in a better way. In the society, persons with nearly equal status, tend to be friendly. In an industry, employees with nearly equal powers form association and move closely. Equitability among citizens in terms of wealth, health, status etc is the goal of a democratic nation. In order to study this practical concept, a graph model is to be created. Prof. E. Sampathkumar is the first person to recognize the spirit and power of this concept and introduced various types of equitability in graphs like degree equitability, outward equitability, inward equitability, equitability in terms of number of equal degree neighbours, or in terms of number of strong degree neighbours etc. In general, if $G=(V, E)$ is a simple graph and $\phi: V(G) \rightarrow N$ is a function, we may define equitability of vertices in terms of $\phi$ - values of the vertices.A wheel graph of order $\mathrm{n}$ is denoted by $W_{n}$. In this graph, one vertex lines at the centre of a circle (wheel) and $n-1$ vertical lies on the circumference. In this Paper, a study is made of equitability defined by degree function.

Definition 1.1. A subset $D$ of $V$ is called an equitable dominating set if for every $v \in V-D$ there exists a vertex $u \in D$ such that $u v \in E(G)$ and $|\operatorname{deg}(u)-\operatorname{deg}(v)| \leq 1$. The minimum cardinality of such a dominating set is denoted by $\gamma^{e}$ and is called the equitable domination number of $G$.

Definition 1.2. A vertex $u \in V$ is said to be degree equitable with a vertex $v \in V$ if $|\operatorname{deg}(u)-\operatorname{deg}(v)| \leq 1$.

Remark 1.1. If $D$ is an equitable dominating set then any super set of $D$ is an equitable dominating set.

Definition 1.3. An equitable dominating set $D$ is said to be a minimal equitable dominating set if no proper subset of $D$ is an equitable dominating set.

Definition 1.4. A minimal equitable dominating set of maximum cardinality is called a $\Gamma^{e}$-set and its cardinality is denoted by $\Gamma^{e}$.

Definition 1.5. An equitable dominating set is said to be 1 - minimal if $D-v$ is not an equitable dominating set for all $v \in D$. 
Remark 1.2. If a vertex $u \in V$ be such that $|\operatorname{deg}(u)-\operatorname{deg}(v)| \geq 2$ for all $v \in N(u)$ then $u$ is in every equitable dominating set. Such vertices are called equitable isolates. Let $I_{e}$ denote the set of all equitable isolates. Vacuously isolated vertices are equitable isolated vertices. Hence $I_{s} \subseteq I_{e} \subseteq$ $D$ for every equitable dominating set $D$ where $I_{s}$ is the set of all isolated vertices of $G$.

Remark 1.3. An equitable dominating set $D$ is minimal if and only if it is 1 - minimal.

\section{Equitable Graph of a Graph}

Definition 2.1. Let $u \in V$. The equitable neighbourhood of $u$ denoted by $N^{e}(u)$ is defined as $N^{e}(u)=\{v \in V / v \in N(u),|d(u)-d(v)| \leq 1\}$ and $u \in I_{e} \Leftrightarrow N^{e}(u)=\phi$.

The cardinality of $N^{e}(u)$ is called equitable degree of $u$ and it is denoted by $d_{G}^{e}(u)$.

Definition 2.2. The maximum and minimum equitable degree of a vertex in $G$ are denoted respectively by $\Delta^{e}(G)$ and $\delta^{e}(G)$. That is $\Delta^{e}(G)=$ $\max _{u \in V(G)}\left|N^{e}(u)\right|$

$\delta^{e}(G)=\min _{u \in V(G)}\left|N^{e}(u)\right|$

Definition 2.3. A subset $S$ of $V$ is called an equitable independent set, if for any $u \in S, v \notin N^{e}(u)$ for all $v \in S-\{u\}$.

Definition 2.4. Let $u \in V(G)$. Then the number of vertices which are degree equitable with $u$, is called degree equitable number of $u$ and is denoted by de(u).

That is $d e(u)=|\{v \in V-\{u\} /|d(v)-d(u)| \leq 1\}|$

Remark 2.1. (i) $d_{G}^{e}(u)+d_{\bar{G}}^{e}(u)=d e(u)$

(ii) $d e_{G}(u)=d e_{\bar{G}}(u) \leq n-1$

Definition 2.5. Let $G=(V, E)$ be a graph. The equitable graph $G^{e}$ of $G$ is defined as the graph with vertex set $V(G)$ and two vertices $u, v$ are adjacent if and only if $u$ and $v$ are degree equitable. 
Remark 2.2. i) If $G$ is regular, then $G^{e}=K_{n}$.

ii) If $G$ is bi-regular graph of biregularity $(r, r+1)$ then $G^{e}=K_{n}$

iii) $\left(K_{1, n}\right)^{e}= \begin{cases}K_{n} \cup K_{1} & \text { if } n \geq 3 \\ K_{n+1} & \text { if } n \leq 2\end{cases}$

iv) $\left(K_{m, n}\right)^{e}= \begin{cases}K_{m+n} & \text { if }|m-n| \leq 1 \\ K_{m} \cup K_{n} & \text { if }|m-n|>1\end{cases}$

v) $\left(P_{n}\right)^{e}=\left(C_{n}\right)^{e}=K_{n}$

vi) $\left(W_{n}\right)^{e}=\left\{\begin{array}{ll}K_{n} & \text { if } n=4,5 \\ K_{n} \cup K_{1} & \text { if } n \geq 6\end{array}\right.$.

vii) $G$ is either regular or bi regular with bi regularity, $(r, r+1)$ if and only if $G^{e}=K_{n}$.

Theorem 2.1. For any graph $G, \gamma\left(G^{e}\right) \leq \gamma^{e}(G)$.

\section{Proof}

Since any equitable dominating set of $\mathrm{G}$ is a dominating set of $G^{e}, \gamma\left(G^{e}\right) \leq$ $\gamma^{e}(G)$.

Theorem 2.2. Let $G$ be a graph such that the set of equitable vertices of $u$ is contained in $N(u)$ for all $u \in V$. (That is the set of equitable vertices of $u$ in $G$ is contained in $\left.V-N_{\bar{G}}[u]\right)$. Then $\gamma\left(G^{e}\right)=\gamma^{e}(G)$.

\section{Proof}

Let $D$ be any $\gamma$-set of $G^{e}$. Then for any $u \in V-D$ there exists $v \in D$ such that $u$ and $v$ are adjacent in $G^{e}$. That is $u$ and $v$ are equitable in $G$. By hypothesis, $u \in N(v)$. Therefore $D$ is an equitable dominating set of $G$. Therefore $\gamma^{e}(G) \leq|D|=\gamma\left(G^{e}\right)$. But $\gamma\left(G^{e}\right) \leq \gamma^{e}(G)$. Therefore $\gamma\left(G^{e}\right)=\gamma^{e}(G)$.

Example 2.1. Let $G$ be the graph obtained by joining a vertex of $K_{n}(n \geq$ $6)$ with a vertex of $K_{m}(m \leq n-3)$. Then $G$ is connected. For any vertex $u$ of $G$, the vertex which are degree equitable with $u$ are in $N(u)$. Also $G$ is not complete. 


\section{Illustration 2.2.}

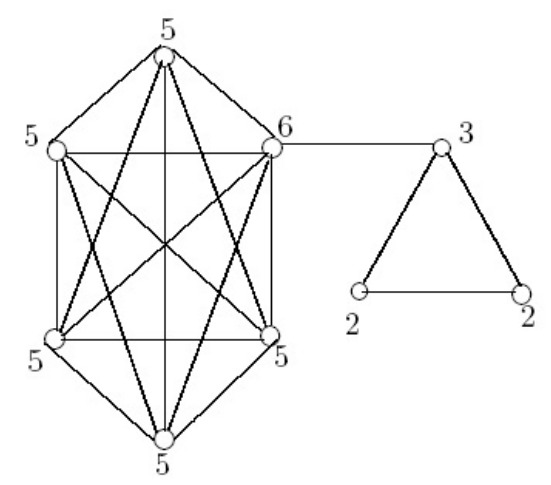

Fig. 2.1

\section{Example 2.3.}

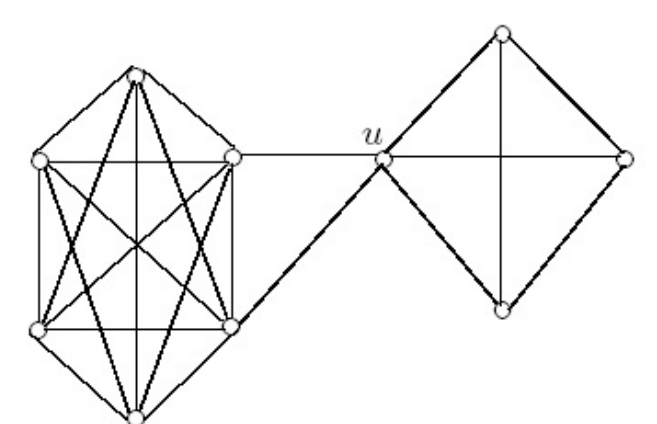

Fig. 2.2

$G^{e}=K_{7} \cup K_{3}$

$\gamma\left(G^{e}\right)=2$. Therefore $\gamma^{e}(G)=\gamma\left(G^{e}\right)$.

But degree equitable vertices of $u$ are not completely connected in $N(u)$. Hence the converse of the previous theorem is not true.

Remark 2.3. There exists a graph $G$ satisfying the following: 
There exists a $\gamma$-set $D$ of $G^{e}$ such that for some vertex $u \in V-D, u$ is not adjacent to any vertex of $D$ in $G$. For example, let $G=K_{6} \cup K_{5}$, $G^{e}=K_{11}$. Let $u \in V\left(K_{6}\right)$ in $G$. Then $D=\{u\}$ is a dominating set of $G^{e}$. Any vertex $v \in V\left(K_{5}\right)$ in $G$ is in $V-D$ and $v$ is not adjacent to any vertex of D. Here $\gamma^{e}(G)=2, \gamma\left(G^{e}\right)=1$.

Remark 2.4. Given any positive integer $r$, there exists an infinite family of graphs for which $\gamma^{e}(G)-\gamma\left(G^{e}\right)=r$. For : Let $G=K_{n} \cup r K_{n-1}(n \geq 2)$. Then $G^{e}=K_{(r+1) n-r} \cdot \gamma^{e}(G)=r+1, \gamma\left(G^{e}\right)=1 . \gamma^{e}(G)-\gamma\left(G^{e}\right)=r$.

Theorem 2.3. $\gamma\left(G^{e}\right)<\gamma^{e}(G)$ if and only if for any $\gamma$ - set $D$ of $G^{e}$ there exists a vertex $u$ in $V-D$ such that $N_{G}^{e}(u) \cap D=\phi$.

\section{Proof}

Assume that for any $\gamma$-set $D$ of $G^{e}$, there exists a vertex $u$ in $V-D$ such that $N_{G}^{e}(u) \cap D=\phi$. Suppose $\gamma\left(G^{e}\right)=\gamma^{e}(G)$. Let $D$ be a $\gamma^{e}$-set of $G$. Then $D$ is a $\gamma$-set of $G^{e}$.

By hypothesis, there exists $u \in V-D$ such that $N_{G}^{e}(u) \cap D=\phi$

which is a contradiction since $D$ is a equitable dominating set of $G$.

Therefore $\gamma\left(G^{e}\right)<\gamma^{e}(G)$.

Conversely, let $\gamma\left(G^{e}\right)<\gamma^{e}(G)$. Suppose the result is not true. Then there exists a $\gamma$-set say $D$ of $G^{e}$ such that for every $u$ in $V-D, N_{G}^{e}(u) \cap$ $D \neq \phi$. Therefore $D$ is an equitable dominating set of $G$. Therefore $\gamma^{e}(G) \leq|D|=\gamma\left(G^{e}\right)$, a contradiction. Hence the theorem.

Remark 2.5. The above theorem can be restated as follows:

$\gamma\left(G^{e}\right)=\gamma^{e}(G)$ if and only if there exists a $\gamma$ set $D$ of $G^{e}$ such that for every vertex $u \in V-D, N_{G}^{e}(u) \cap D \neq \phi$.

Theorem 2.4. Given any positive integer $k$, there exists a graph $G$ such that $\gamma^{e}(G)-\gamma(G)=k$.

For: Consider $K_{1, k}(k \geq 3)$

$$
\gamma^{e}\left(K_{1, k}\right)=k+1, \gamma\left(K_{1, k}\right)=1 \text {. Therefore } \gamma^{e}\left(K_{1, k}\right)-\gamma\left(K_{1, k}\right)=k
$$


Theorem 2.5. $\gamma(G)=\gamma^{e}(G)$ if and only if there exists a $\gamma$-set $D$ of $G$ such that for every vertex $u \in V-D, N_{G}^{e}(u) \cap D \neq \phi$.

\section{Proof}

Assume that $\gamma(G)=\gamma^{e}(G)$. Suppose the result is not true. Then for any $\gamma$-set $D$ of $G$ there exists a vertex $u \in V-D$ such that $N_{G}^{e}(u) \cap D=\phi$. Let $D$ be a $\gamma^{e}$-set of $G$. Then $D$ is a dominating set of $G$ with $|D|=\gamma^{e}=\gamma$. Therefore $D$ is a $\gamma$-set of $G$. Therefore by our assumption, there exists $u \in V-D$ such that $N_{G}^{e}(u) \cap D=\phi$, a contradiction, since $D$ is an equitable dominating set of $G$. Hence the result is true.

Conversely, let the result be true. Then there exists a $\gamma$-set $D$ of $G$ such that for every vertex $u \in V-D, N_{G}^{e}(u) \cap D \neq \phi$. Therefore $D$ is an equitable dominating set. Therefore $\gamma^{e}(G) \leq|D|=\gamma(G)$. But $\gamma \leq \gamma^{e}$. Therefore $\gamma=\gamma^{e}$.

Remark 2.6. The following are infinite classes of graphs which satisfy $\gamma=\gamma^{e}$.

(i) All regular graphs.

(ii) All bi regular graphs with bi regularity $(k, k+1)$.

(iii) Join a vertex of $K_{n}$ to a vertex of $K_{n-2}$. The resulting graph $G$ is neither regular nor bi regular, but $\gamma(G)=\gamma^{e}(G)=2$.

\section{Illustration 2.4 .}

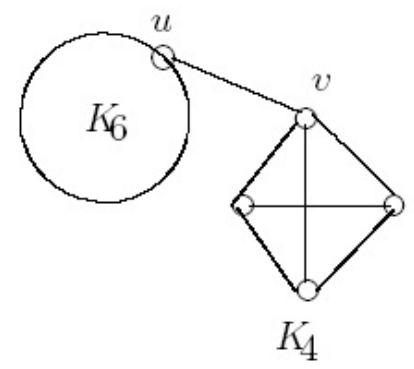

Fig. 2.3

$\{u, v\}$ is both a $\gamma^{e}$-set and a $\gamma$-set.

(iv) Join a vertex $K_{n-2}$ to any two vertices of $K_{n}$. The resulting graph $G$ 
is neither regular nor bi regular, but $\gamma(G)=\gamma^{e}(G)=2$.

\section{Illustration 2.5}

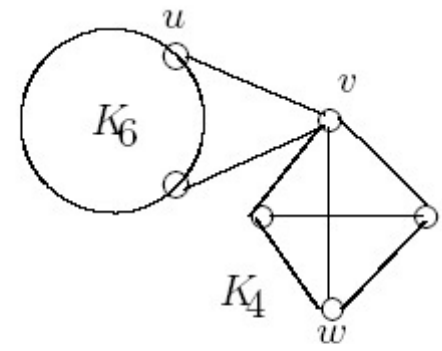

Fig. 2.4

$\{u, w\}$ is both a $\gamma^{e}$-set and a $\gamma$-set.

(v) Let $G$ :

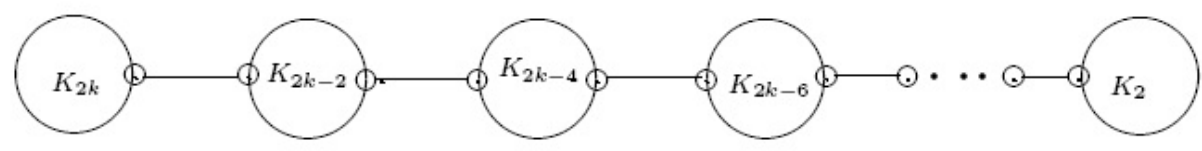

Fig. 2.5

For this graph, $\gamma(G)=\gamma^{e}(G)=k$. 
Remark 2.7. Given any positive integer $k$, there exists an infinite family of non regular, non bi regular graphs with $\gamma(G)=\gamma^{e}(G)=k$. For consider $K_{1}, K_{d+1}, K_{2 d+1}, \ldots, K_{(k-1) d+1}$ where $d$ is any positive integer. Join a vertex of $K_{i d+1}$ with a vertex of $K_{(i+1) d+1}, 0 \leq i \leq k-2$. Let $G$ be the resulting graph. Then $\gamma(G)=\gamma^{e}(G)=k$. Thus we have an infinite family of graphs which are neither regular nor bi regular satisfying the property $\gamma=\gamma^{e}=k$.

\section{References}

[1] R. B. Allan and R. C. Laskar, On domination and independent domination numbers of a graph, Discrete Math. 23, pp. 73-78, (1978).

[2] D. W. Bange, A. E. Barkankas, L. H. Host and P. J. Slater, Generalised domination and domination in graphs, Discrete Math. 159, pp. 1-11, (1996).

[3] C. Berge, Theory of graphs and its applications, Dunod, Paris, (1958).

[4] C. Berge, Graphs and Hypergraphs, North-Holland, Amsterdam, (1973).

[5] C. Berge, and P. Duchet, Recent problem and results about kernels in directed graphs, Discrete Math. 86, pp. 27-31, (1990).

[6] A. P. Burger, E. J. Cockayne and C. M. Mynhardt, Domination Numbers for the Queen's graph, Bull. Inst. Comb. Appl.10, pp. 73-82, (1994).

[7] E. J. Cockayne, S. T. Hedetniemi, Towards a theory of domination in Graphs, Networks 7, pp. 247-261, (1977).

[8] E. J. Cockayne, R. M. Dawes and S. T. Hedetniemi, Total domination in Graphs, 10, pp. 211-219, (1980).

[9] E. J. Cockayne, C. W. Ko, and F. B. Shepherd, Inequalities Concerning dominating sets in graphs, Technical Report DM-370-IR, Dept. Math., Univ. Victoria, (1985).

[10] Venkatasubramanian Swaminathan and Kuppusamy Markandan Dharmalingam Degree Equitable Domination on Graphs,Kragujevac journal of Mathematics, Volume 35, Number 1, pp. 177 - 183, (2011). 


\section{Kuppusamy Markandan Dharmalingam}

Department of Mathematics,

The Madura College,

Madurai- 625011 ,

India

e-mail : kmdharma6902@yahoo.in 